\title{
Aplicativos de smartphones e atividades físicas: contribuições e limitações
}

\section{Smartphone applications and physical activities: contributions and limitations}

\section{Aplicaciones de smartphone y actividades físicas: contribuciones y limitaciones}

\author{
iD (9) Renato Henrique Verzani \\ Universidade Estadual Paulista, Rio Claro, São Paulo, Brasil. \\ renato_verzani@hotmail.com \\ iD Adriane Beatriz de Souza Serapião \\ Universidade Estadual Paulista, Rio Claro, São Paulo, Brasil. \\ adriane@rc.unesp.br
}

Resumo: A ampliação da utilização de smartphones e dos recursos dos aplicativos pode trazer contribuições para a diminuição de fatores de risco à saúde em nível mundial, como os da inatividade física. Sendo assim, o objetivo deste artigo foi analisar as evidências sobre o uso de aplicativos na atividade física, buscando compreender potencialidades, limitações e estratégias motivacionais envolvidas. Por meio de uma revisão narrativa, constataram-se diversas funcionalidades e estratégias que podem contribuir tanto para promover um estilo de vida ativo quanto para o acompanhamento de condições crônicas, favorecendo assim a intervenção profissional. Por outro lado, o respeito a diretrizes e outros fatores apontados revelaram a necessidade de cuidados, além da conscientização e da educação dos usuários, minimizando riscos e viabilizando resultados mais positivos.

Palavras-chave: Educação Física e treinamento. Desenvolvimento Tecnológico. Estratégias de eSaúde. 
Abstract: The expansion in the use of smartphones and the resources of applications can bring contributions to risk factors, such as physical inactivity. Therefore, the aim was to analyze the evidence on the use of applications in physical activity, seeking to understand the potential, limitations and motivational strategies involved. Through a narrative review, several functionalities and strategies were found that can contribute both to an active lifestyle and to chronic conditions, contributing to professional intervention. However, respect for guidelines and other factors pointed out reveals the need for care, in addition to the need for awareness and education, minimizing risks and enabling positive results.

Keywords: Physical Education and Training. Technological Development. eHealth Strategies.

Resumen: La expansión en el uso de teléfonos inteligentes y los recursos de las aplicaciones puede aportar contribuciones a los factores de riesgo, como la inactividad física. Por lo tanto, el objetivo era analizar la evidencia sobre el uso de aplicaciones en la actividad física, buscand o comprender las potencialidades, limitaciones y estrategias motivacionales relacionadas. A partir de una revisión narrativa, se encontraron varias funcionalidades y estrategias que pueden contribuir tanto a un estilo de vida activo como a condiciones crónicas, lo que contribuye a la intervención profesional. Sin embargo, el respeto a las pautas y otros factores señalados revela la necesidad de atención, concienciación y educación, minimizando los riesgos y permitiendo resultados positivos.

Palabras-clave: Educación y Entrenamiento Físico. Desarrollo Tecnológico. Estrategias de eSalud.

Submetido em: 19-07-2019

Aceito em: 06-07-2020 
Aplicativos de smartphones e atividades físicas: contribuições e limitações

Renato Henrique Verzani • Adriane Beatriz de Souza Serapião

\section{Introdução}

A crescente e ampla utilização de smartphones nos últimos anos permitiu algumas alterações importantes em hábitos e comportamentos, como a possibilidade de as pessoas acessarem facilmente muitas informações sobre saúde, considerando desde os aplicativos que atendem a esta demanda, até mesmo a sites e redes sociais virtuais (HASMAN, 2011).

Middelweerd et al. (2014) salientam a importância de as pessoas estarem sempre portando seus smartphones e acessando informações em qualquer lugar e momento. Assim, aplicativos que visem à promoção de comportamentos adequados quanto à atividade física poderiam oferecer informações e aconselhamentos individualizados nos momentos mais adequados.

Para Yuan et al. (2015), um dos pontos fortes da utilização destes aplicativos de saúde e fitness estaria no monitoramento de questões de saúde do usuário e também no gerenciamento de questões individuais de saúde. Assim, os aplicativos móveis seriam software planejados para uso em dispositivos móveis, como os smartphones.

Gabbiadini e Greitemeyer (2019) enfatizam o potencial revolucionário que dispositivos móveis têm, causando mudanças nas comunicações das pessoas. Esses autores destacam dados apontando que aproximadamente seis entre cada 10 usuários de smartphones já baixaram aplicativos relacionados à saúde. Ainda segundo eles, a adoção de um aplicativo fitness pode disponibilizar às pessoas informações que promoveriam uma atitude mais positiva, sendo que esse fato pode ser um preditor de um estilo de vida ativo.

Birkhoff e Smeltzer (2017) citam que existem mais de 150 mil aplicativos relacionados à saúde disponibilizados, com destaque para os de condicionamento físico e dieta. Além disso, afirmam que há uma atenção voltada à criação de aplicativos direcionados 
Aplicativos de smartphones e atividades físicas: contribuições e limitações

Renato Henrique Verzani • Adriane Beatriz de Souza Serapião

a condições crônicas, tais como as relacionadas à saúde cardiovascular e diabetes, por exemplo.

Desta maneira, a centralização no indivíduo permitida pelos aplicativos de saúde móvel tem relação direta com sua popularidade (BIRKHOFF; SMELTZER, 2017). Sarkar et al. (2016) apontam para a importância da atenção à saúde e o gerenciamento das condições crônicas, já Birkhoff e Moriarty (2016) citam a capacitação para que os usuários contribuam com sua saúde e busca pelo bem-estar. Assim, existe uma expectativa de que tenha havido um crescimento de quase 50\% entre os anos de 2014 e 2020 nos serviços de monitoramento por meio dos smartphones (GRAND VIEW RESEARCH, 2015 apud BIRKHOFF; SMELTZER, 2017) e de um mercado da ordem de 151,57 bilhões de dólares até 2025 (GRAND VIEW RESEARCH, 2018).

O aumento no uso de smartphones e também dos aplicativos voltados à saúde criou novas possibilidades e alterações na perspectiva de monitoramento e motivação dos usuários (HIGGINS, 2016). Grande parte dos aplicativos mais usados na área de saúde e atividade física tem o foco no condicionamento e também no automonitoramento (SAMA et al., 2014). Muitos deles podem se tornar uma ferramenta muito importante para auxiliar os profissionais da área no acompanhamento dos parâmetros de saúde, nas metas que podem ser traçadas e buscadas, além de auxiliar pacientes que precisam atingir objetivos determinados (HIGGINS, 2016).

Os aplicativos de atividades físicas normalmente contam com rastreamento via GPS (sistema de posicionamento global) dos registros das atividades realizadas, como no caso de corridas, de caminhadas ou ciclismo. Além disso, outros parâmetros são registrados, como frequência, intensidade e duração da atividade, possibilitados por giroscópios e/ou acelerômetros (WU et al., 2012). Também é comum encontrar estimativas de gasto calórico, resumos de tendências de performance em certos períodos de tempo, além de permitir o compartilhamento desses dados nas redes sociais virtuais (WANG et al., 2016). 
Aplicativos de smartphones e atividades físicas: contribuições e limitações

Renato Henrique Verzani • Adriane Beatriz de Souza Serapião

Além das possibilidades anteriores, Bort-Roig et al. (2014) citam a utilização de câmeras, autofalante e microfones, permitindo à tecnologia dos smartphones uma boa precisão relacionada às informações sobre atividades físicas, podendo inclusive dinamizar e diversificar algumas práticas. Com isso, por meio das tecnologias disponibilizadas, os aplicativos podem monitorar diferentes parâmetros biológicos, envolvendo atividade física, reabilitação cardíaca, quedas de idosos, dentre outros (RECIO-RODRIGUEZ et al., 2014).

Existe uma grande diversidade de aplicativos voltados ao condicionamento físico e à saúde que incluem: busca por melhorias nas atividades físicas realizadas, estabelecimento de metas de peso, alimentação, controle do sono, relaxamento, entre outras (HIGGINS, 2016). Outra potencialidade destes recursos é a sincronização sem fio com outras tecnologias, como os dispositivos vestíveis (pulseira, frequencímetro, roupas inteligentes etc.) (HIGGINS, 2016), ampliando e potencializando assim as possibilidades de registro das informações.

Abre-se espaço então para que, os avanços nas tecnologias móveis possam contribuir com questões como a inatividade física e os problemas globais a ela associados (MATTHEWS et al., 2016). A evolução desse campo e o acesso à Internet crescendo em nível mundial dão à saúde móvel um papel fundamental na educação em saúde nos próximos anos, podendo transformar as intervenções dos profissionais da área (BOULOS et al., 2011).

Sendo assim, o objetivo geral deste estudo foi analisar as evidências sobre o uso de aplicativos na atividade física. Como objetivos específicos, buscou-se explicitar as potencialidades dos aplicativos no combate à inatividade física, compreender quais os riscos e limitações dessas ferramentas na atualidade e entender as estratégias utilizadas para manter a motivação dos usuários.

Como justificativa do estudo, consideramos a imersão cada vez mais representativa das pessoas em tecnologias como smartphones (HASMAN, 2011), as possibilidades voltadas ao monitoramento 
Aplicativos de smartphones e atividades físicas: contribuições e limitações

Renato Henrique Verzani • Adriane Beatriz de Souza Serapião

da saúde por meio de aplicativos e seus possíveis reflexos positivos ao usuário (GABBIADINI; GREITEMEYER, 2019) e ainda a viabilidade da inclusão destas ferramentas na atuação de profissionais da saúde (HIGGINS, 2016). É fundamental também compreender quais são os seus avanços e as suas potencialidades, bem como os possíveis cuidados e lacunas que ainda precisam ser preenchidas para uma utilização cada vez mais segura por parte de usuários e profissionais, permitindo assim a visualização de um panorama atualizado desta nova realidade.

Foi realizada uma revisão narrativa, que como destaca Rother (2007), é adequada à descrição e à discussão do "estado de arte" de uma temática, permitindo assim obter ou atualizar o conhecimento sobre a mesma, sem apresentar uma metodologia rigorosa que viabilize a reprodução dos dados e também sem respostas quantitativas para determinadas questões específicas. Também neste sentido, Elias et al. (2012) relatam a busca de interpretações críticas mais amplas, contribuindo para o entendimento deste "estado de arte", bem como viabilizando novas perspectivas. Já para Vosgerau e Romanowski (2014), estado de arte e estado de conhecimento têm aparecido como semelhantes nas investigações, sendo que nesse sentido não basta a identificação de produção, mas sim evidenciar as diferentes perspectivas e enfoques, situando o tema na atualidade.

Com base nestas informações, a busca ocorreu em artigos, dissertações, teses e documentos de instituições diretamente envolvidas com a temática, analisando o impacto que podem ter na saúde e nas complicações relacionadas com a inatividade física. Foram utilizadas as palavras-chave "aplicativos", "smartphones", "atividades físicas", "inatividade física" e "mHealth" nas bases de dados Pubmed, Scielo, Lilacs, Periódicos Capes e Google Scholar.

O critério de inclusão era apresentar as palavras buscadas no título, nas palavras-chave, ou claramente pelo resumo constatar que era condizente com a temática em questão. Como critério de exclusão, foram considerados os artigos em duplicidade, os que tratavam apenas de questões técnicas relacionadas ao desenvolvi- 
Aplicativos de smartphones e atividades físicas: contribuições e limitações

Renato Henrique Verzani • Adriane Beatriz de Souza Serapião

mento de aplicativos e os que não tratavam da temática proposta. Atendendo aos objetivos traçados, as próximas seções discutem o atual panorama envolvendo a utilização destas tecnologias, potencialidades, estratégias motivacionais relacionadas, limitações e as considerações finais sobre o tema.

\section{Combate à inatividade física e tecnologias: abertura de novos caminhos?}

Considerando a inatividade física e a falta de condicionamento, temos dados da Organização Mundial da Saúde (OMS) que apontam para cerca de $30 \%$ dos adultos com níveis insuficientes de atividades (HALLAL et al., 2012; HIGGINS, 2016). A prática de atividades físicas insuficientes é um dos principais fatores de risco de morte em nível mundial, sendo que um fato preocupante é que mais de $80 \%$ dos adolescentes se enquadram nesse nível insatisfatório de atividade física (WHO, 2018).

Pesquisas têm apontado que níveis inadequados de atividade física contribuem significativamente para obesidade, doenças crônicas e também aumentam a mortalidade (REZENDE et al., 2014). É preciso prestar atenção ao fato de que a inatividade física é um problema de ordem mundial (HALLAL et al., 2012; ANDERSEN; MOTA; DI PIETRO, 2016). Existem estimativas de aumento em torno de 20 a 30\% no risco de mortalidade ligada à inatividade física (BISWAS et al., 2015). Sendo assim, é importante aumentar a participação regular em atividades e exercícios físicos, permitindo o controle do peso corporal e também diminuindo riscos de diversas comorbidades (REINER et al., 2013). Alterar questões relacionadas ao estilo de vida das pessoas é importante para gerenciar as doenças crônicas (MATTHEWS et al., 2016).

Dentre essas alterações, a inserção da atividade física é bastante eficaz na diminuição das taxas de internações hospitalares e também do risco de mortalidade (MATTHEWS et al., 2016). 
Aplicativos de smartphones e atividades físicas: contribuições e limitações

Renato Henrique Verzani • Adriane Beatriz de Souza Serapião

Concordando com esses autores, Arem et al. (2015) destacam que o aumento nos níveis de atividades físicas tem enorme potencial de redução da morte prematura, bem como no prolongamento da vida saudável. Níveis elevados de atividade física, com intensidades moderadas, têm se demonstrado um fator que diminui o risco de mortalidade ligada ao sedentarismo (EKELUND et al., 2016). Esses fatos confirmam a necessidade de aumentar os estímulos para as atividades físicas, minimizando as doenças e as mortes prematuras (GAL et al., 2018).

Por outro lado, é uma realidade que não é fácil chegar a pessoas sedentárias e promover mudanças de comportamento relacionado à atividade física, bem como manutenção deste novo hábito (SAMDAL et al., 2017). Contudo, os avanços tecnológicos, envolvendo tanto os smartphones quanto outros dispositivos, passaram a ser grandes aliados visando a intervenções nas atividades físicas (SANDERS et al., 2016).

Diversos dispositivos vestíveis (wearables) representam uma maneira simples e atraente visando ao automonitoramento das atividades físicas (RABIN; BOCK, 2011). Aliados aos avanços dos aplicativos nos smartphones, é viável a utilização e combinação de inúmeras técnicas que visem mudar o comportamento e promover a atividade física (RABIN; BOCK, 2011; MIDDELWEERD et al., 2014).

Esse tipo de autogerenciamento dos usuários, aliado às tecnologias, pode trazer alterações valiosas no engajamento, ampliando a atratividade e tornando-os mais envolvidos com os próprios resultados (KNIGHT, 2012). Os aplicativos e os wearables desenvolvidos permitem o automonitoramento e os feedbacks (SANDERS et al., 2016; MCCALLUM; ROOKSBY; GRAY, 2018), melhorando então os níveis de atividades físicas e os resultados para a saúde (HICKEY; FREEDSON, 2016).

Visando contribuir para mudanças nos comportamentos de usuários em atividades físicas, existem diversas técnicas, sendo que a do automonitoramento é bem eficaz e importante (MURRAY et al., 2017). É possível ampliar a sua contribuição a partir da as- 
Aplicativos de smartphones e atividades físicas: contribuições e limitações

Renato Henrique Verzani • Adriane Beatriz de Souza Serapião

sociação com outras técnicas, como a do feedback, a de formação imediata de intenção, a do estabelecimento de metas, dentre outras (DIREITO et al., 2014).

McCallum, Rooksby e Gray (2018) destacam a ascensão do interesse nas pesquisas envolvendo o impacto dos wearables e dos aplicativos de atividades físicas nos últimos anos. Uma revisão sobre intervenções voltadas às atividades físicas baseadas na Internet expôs que aproximadamente 60\% dos estudos apresentaram contribuições significativas (JOSEPH et al., 2014). Knight et al. (2015) salientam que a conexão com dispositivos voltados à saúde pode trazer resultados clínicos positivos, bem como intervenções que aliam dispositivos móveis e exercícios físicos têm apresentado resultados importantes nos marcadores de saúde cardiovascular. Desta maneira, fica bastante evidente que os aplicativos e recursos tecnológicos possuem um potencial muito grande dentro da área de atividades e exercícios físicos.

\section{Recursos e potencialidades}

De acordo com Demonte (2016), as aplicações do mHealth foram impulsionadas pela expansão da utilização de dispositivos móveis touch, dando espaço então para aplicativos que monitoram questões relacionadas à saúde, tanto coletiva quanto individualmente. Esta autora cita que, de acordo com um relatório disponibilizado no ano de 2014, existem 5 grandes grupos de aplicativos mHealth: fitness, referências médicas (caráter instrutivo), bem-estar, nutrição e gestão de doenças.

Os aplicativos de exercícios monitoram atividades físicas, sendo encontrados os voltados aos aeróbios, à musculação e também ao alongamento (DEMONTE, 2016). Segundo ela, algumas das funcionalidades comuns são: perfil online com feed de atividades; personalização de objetivos, metas, treinos; treinos ou desafios pré-cadastrados; acompanhamento de gastos calóricos; conexão com 
Aplicativos de smartphones e atividades físicas: contribuições e limitações

Renato Henrique Verzani • Adriane Beatriz de Souza Serapião

redes sociais virtuais; controle de música; feedback por áudio e/ou cronometragem por áudio; feedback de desempenho, progresso e histórico de atividades; medição de batimentos cardíacos; sistema de premiações e/ou quebra de recordes; vídeos de treinamentos; dentre diversos outros recursos.

Melo (2017) destaca que esses tipos de aplicativos têm como foco principal o registro de dados envolvendo as práticas esportivas dos usuários, como velocidade, distância, número de passos, estimativas de calorias, dentre outros. Além disso, também relata que são compatíveis muitas vezes com recursos de monitoramento das atividades físicas, além de estimular a competitividade entre os usuários.

A associação entre os aplicativos e os wearables possibilitada pelo desenvolvimento tecnológico dos últimos anos permitiu um conhecimento maior dos usuários quanto às suas atividades realizadas, quantificando e detalhando as informações referentes aos exercícios (PATEL; ASCH; VOLPP, 2015). Desta maneira, é viável receber feedback sobre prática por meio de resumos e análises dos dados, sendo que alguns aplicativos só permitem a sincronização com os dados de sua marca de wearables e outros permitem acesso aos dados de várias marcas diferentes (STRAGIER; ABEELE; MAREZ, 2018).

Algumas coisas interessantes que podem ser buscadas em aplicativos da área são destacadas por Higgins (2016), como: apresentar interface simples; ter versão gratuita para avaliação; ser de instalação simples e confiável na utilização; ter possibilidade de definição de metas; possuir feedback individualizado; ser personalizado para aptidão do usuário; ter especialistas disponíveis para consulta; utilizar técnicas de mudança de comportamentos embasadas em evidências; possibilitar a sincronização com outros aplicativos e dispositivos; permitir a ligação com redes sociais e, por fim, disponibilizar periodicamente resumos.

Uma possibilidade que pode ser bem aproveitada nesses recursos é o compartilhamento de resultados e conquistas nas re- 
Aplicativos de smartphones e atividades físicas: contribuições e limitações

Renato Henrique Verzani • Adriane Beatriz de Souza Serapião

des sociais virtuais, como Facebook e Instagram (HIGGINS, 2016). Considerando que uma das barreiras encontradas para a prática regular de atividade física é a falta de incentivo por parte de amigos e familiares, a chance de utilizar recursos virtuais das redes sociais dos aplicativos que incentivam o compartilhamento destas informações pode ser benéfica para a superação dessa falta de estímulos (NAKHASI et al., 2014).

Knight et al. (2015) também destacam que os recursos sociais em aplicativos de atividades físicas podem auxiliar os usuários no desenvolvimento de comportamentos mais saudáveis, considerando, então, que a sua inclusão aprimora a utilidade dos aplicativos. Para eles, isso se deve ao compartilhamento de informações, ao vínculo com outros praticantes, a acessos a outras redes sociais e a possibilidade de postar os treinos.

No estudo de Wang et al. (2016), os autores apresentam resultados nos quais são levantadas influências qualitativas percebidas pelos usuários dos aplicativos. Neles, quatro temas são destacados nas discussões: influência na ação (alimentação e atividades físicas), influência na autoeducação (nutrição e atividade física; conhecimento e experiência), influência na vida social (avaliação dos outros e aumento da rede de contatos) e influência na autoavaliação e consciência (motivação, consciência e confiança).

Existem apontamentos de que recursos baseados na Internet têm visado à motivação dos usuários em melhorar a aptidão e também que têm apresentado aumento na adesão (SPITTAELS; BOURDEAUDHUIJ; VANDELANOTTE, 2007). Alguns estudos têm apresentado bons resultados em intervenções de perda de peso a partir de dispositivos para monitorar as atividades físicas em relação a não utilização de nenhum monitoramento (SHUGER et al., 2011).

Contudo, alguns autores levantam a dúvida se os aplicativos auxiliam na adesão às práticas recomendadas ou se os praticantes que seguem as recomendações têm maior propensão de utilizar as ferramentas dos dispositivos móveis (TURNER-McGRIEVY et al., 
Aplicativos de smartphones e atividades físicas: contribuições e limitações

Renato Henrique Verzani • Adriane Beatriz de Souza Serapião

2013). Aroni et al. (2017) encontraram dados que demonstram que os usuários de aplicativos têm maiores índices de atividades físicas do que os praticantes que não usavam esses recursos.

Por outro lado, Gal et al. (2018) relatam que a combinação entre recursos vestíveis e aplicativos na promoção de atividades físicas normalmente é acompanhada nos estudos por outros tipos de intervenções, como sessões de aconselhamento. Schoeppe et al. (2016) destacam que apenas disponibilizar aplicativos possui eficiência menor do que incentivar aplicativos que possuem intervenções adicionais. Assim, entre oferecer apenas um aplicativo ou wearable e, além disso, proporcionar intervenções que aliem outros componentes, pode apresentar resultados diferentes, com efeitos mais benéficos no segundo caso (GAL et al., 2018).

Alguns resultados interessantes sobre a utilização dos aplicativos podem ser encontrados na literatura. Wang et al. (2016) observaram em seu estudo que a maioria dos usuários creditou à utilização desses aplicativos uma prática mais frequente de exercícios, bem como o aumento da intensidade e a diversificação das atividades. Ainda de acordo com esse estudo, os autores sugerem que a utilização de aplicativos de atividades físicas e dietas é vista como eficaz pelos usuários, apresentando efeito sobre atitudes, autoeducação e consciência sobre saúde, sobre atividades físicas e nutrição e também acerca da vida social, principalmente quando utilizados por longos períodos. Gabbiadini e Greitemeyer (2019) constataram que um aplicativo de contagem de passos diários pode trazer efeitos positivos na promoção de um comportamento saudável.

Aroni et al. (2017) encontraram como características importantes destacadas pelos usuários de aplicativos: as calorias queimadas, os dados sobre distância percorrida e mapeamento e também as informações referentes à frequência cardíaca. Wang et al. (2016) relataram algumas questões interessantes, como a importância do feedback para melhorar a motivação para prática e a possibilidade dos aplicativos desempenharem o papel de lembrete ou avaliador, sendo que muitas vezes os usuários aumentavam a sua 
Aplicativos de smartphones e atividades físicas: contribuições e limitações

Renato Henrique Verzani • Adriane Beatriz de Souza Serapião

autoconfiança ao visualizarem melhorias na alimentação ou progressos nos exercícios ao longo do tempo.

A análise de Middelweerd et al. (2014) destacou que os aplicativos possuem o potencial de disponibilizar feedback individualizado e contêm técnicas relacionadas com alterações no comportamento. O fornecimento de feedback, o automonitoramento e o estabelecimento de metas, de acordo com estes autores, são utilizados com maior frequência.

O fato é que alguns estudos apresentaram perspectivas positivas quanto ao uso dos aplicativos nas mudanças de comportamentos (HIGGINS, 2016), exemplificando que achados apontam para melhoras significativas na atividade física moderada de adultos que tiveram acesso diariamente ao feedback, além da definição de metas e utilização de recursos móveis. A utilização das metas é considerada muito importante visando à atividade física, sendo que, quando personalizadas, apresentam maior eficácia do que as mais generalistas (LOCKE; LATHAM, 2006). Assim, constatamos que intervenções nas atividades físicas com aplicativos ou recursos vestíveis são bastante promissoras e disponibilizam diversas oportunidades de individualização, aumentando assim a sua eficácia (GAL et al., 2018).

\section{Estratégias utilizadas para aumentar a motivação}

Outra perspectiva quanto à busca por envolvimento dos usuários compreende um conceito que vem sendo bastante utilizado, que é o da gamificação, que seria a utilização descontextualizada de técnicas relacionadas à área dos jogos, visando a colaborar na motivação para envolvimento, engajamento e interação de pessoas, atingindo inclusive áreas como o treinamento e a saúde (LAMBOGLIA et al., 2016). Os autores citam ainda que alguns exemplos desta estratégia são rankings, acúmulos de pontos com possibilidades de trocas por algum tipo de benefício, recompensas, distintivos ou insígnias. 
Aplicativos de smartphones e atividades físicas: contribuições e limitações

Renato Henrique Verzani • Adriane Beatriz de Souza Serapião

Estratégias como da gamificação buscam auxiliar na busca de objetivos por meio da motivação extrínseca (LAMBOGLIA et al., 2016). Contudo, quando são motivadas de modo intrínseco, as pessoas costumam retornar à prática por vontade própria, enquanto quem está motivado extrinsecamente necessita de um estímulo externo para retomar a atividade (RYAN; DECI, 2000a; LAMBOGLIA et al., 2016).

Assim, Lamboglia et al. (2016) levantam a discussão quanto ao sistema de recompensa oferecido pela gamificação e a Teoria da Autodeterminação (RYAN; DECl, 2000b), visto que esta relação poderia reduzir a motivação intrínseca, pois este sistema utiliza-se apenas da motivação extrínseca. Com isso, a realização da tarefa passaria a ser focada na busca pela recompensa, sem levar em conta a sua real motivação (NICHOLSON, 2012; LAMBOGLIA et al., 2016).

Winterstein e Venditti Jr (2009) destacam que o prazer pela atividade está diretamente envolvido com a motivação intrínseca, podendo estar relacionada à autossuperação, ao prazer relacionado à aprendizagem, a vivenciar situações diferentes, dentre outros. Já quando há um direcionamento extrínseco, ainda segundo os autores, o que se busca é o que será conquistado fora, sendo este envolvimento um meio, não um fim.

Cabe salientar também a complementariedade dessas tendências, isto é, elas não são excludentes, mas, sim, há uma ênfase em uma delas (WINTERSTEIN; VENDITTI JR, 2009). Estes autores também citam o cuidado quanto ao uso de recompensas para induzir as pessoas à prática esportiva, devendo-se ter cautela com o abuso da recompensa. Isso se daria pelo fato de que pessoas que estejam envolvidas intrinsecamente e recebem inúmeros estímulos extrínsecos podem alterar a sua fonte de prazer, tendendo a esperar por mais recompensas (WINTERSTEIN; VENDITTI JR, 2009).

Werbach e Hunter (2012) também destacam a questão de influência da motivação intrínseca a partir do recebimento de estímulos extrínsecos. Contudo, estes autores também salientam que 
Aplicativos de smartphones e atividades físicas: contribuições e limitações

Renato Henrique Verzani • Adriane Beatriz de Souza Serapião

a motivação extrínseca possui papel fundamental em tarefas que não motivam, proporcionando bons resultados de desempenho, podendo conduzir ao interesse pela atividade. Assim, consideram também que em tarefas nas quais não há interesse intrínseco, a utilização dessas recompensas poderia viabilizar alterações no comportamento.

Há então uma linha de raciocínio que destaca a possibilidade da motivação extrínseca aproximar o indivíduo da atividade e, a partir de seu envolvimento, pode haver um aumento na autonomia e um incentivo interno (LAMBOGLIA et al., 2016).

Considerando as três necessidades psicológicas básicas (competência, autonomia e pertencimento), Werbach e Hunter (2012) demonstram que a inclusão dessa perspectiva dos jogos permitiria que a competência estivesse ligada à disponibilização de pontuação e níveis, enquanto que as escolhas ao longo do desenvolvimento corresponderiam à autonomia, tendo, posteriormente, o compartilhamento em redes sociais virtuais de informações e conquistas relação com o pertencimento.

Desta maneira, a gamificação estaria relacionada com a motivação, o incentivo e a interação com o usuário (LAMBOGLIA et al., 2016). Ainda de acordo com os autores, a relação da motivação das pessoas com a autoeficácia, aprovação social e domínio permite a utilização das recompensas, da competição e da atenção.

Os mHealths, ou aplicativos móveis de saúde, podem ser vistos como ferramentas com ótimo potencial de popularização, visto que há uma sinalização de que são de baixo custo, facilmente acessíveis, atrativos e também contam com boa aceitação do público (LAMBOGLIA et al., 2016). Estes autores destacam os mHealths como uma inovação que pode ser uma importante aliada tanto na prevenção como no combate a inúmeras doenças, salientando a possibilidade de aproximar as pessoas dos profissionais da saúde, disponibilizando informações que permitem a tomada de decisões mais rápidas pelo caráter atualizado das mesmas, já que são disponibilizadas em tempo real, criando assim resultados mais positivos para a saúde do usuário. 


\section{Cuidados envolvendo a utilização}

Alguns pontos que podem representar limitações aos aplicativos também foram apontados. Segundo Higgins (2016), vários fatores podem comprometer os resultados no seu uso como: ter um aparelho com acesso a plano de dados, a falta de revisão por profissionais devidamente treinados, a necessidade de estar com o smartphone na atividade (que pode ser inconveniente pelo tamanho, risco de molhar com água ou suor), a falta de embasamento em evidências ou teorias de mudanças de comportamentos, a imprecisão dos sensores do smartphones em relação a sensores específicos e as questões relacionadas à sincronização de alguns aplicativos, além de possíveis problemas técnicos ou mau funcionamento.

Além disso, alguns cuidados precisam ser tomados na escoIha e na utilização dos aplicativos. Segundo Modave et al. (2015), o American College of Sports Medicine (ACSM) possui recomendações voltadas aos programas de exercícios com base na intensidade, frequência, tempo e tipo, necessitando de progressões de acordo com o condicionamento do indivíduo e também com base em metas específicas de saúde.

Por outro lado, segundo o que apresentam no estudo, ao revisar alguns aplicativos, constataram que nenhum dos aplicativos analisados atendia as diretrizes preconizadas pelo ACSM (MODAVE et al., 2015). Assim, estes autores afirmam que, apesar da grande disponibilidade, poucos aplicativos apresentam qualidade para prescrição, principalmente para iniciantes. Destacam ainda que há risco de engajamento em exercícios sem o nível esperado ou inadequado à preparação técnica e física do usuário.

Outro estudo, de Herrmann e Kim (2017), também trata desta temática. Nesse trabalho os autores abordam problemas relacionados a inconsistências quanto às diretrizes de atividade física que podem também trazer problemas no estabelecimento de metas por não estarem baseadas no que seria realmente adequado. No 
Aplicativos de smartphones e atividades físicas: contribuições e limitações

Renato Henrique Verzani • Adriane Beatriz de Souza Serapião

estudo de Knight et al. (2015) não foram encontrados aplicativos para atividades aeróbicas com metas de saúde pública baseadas em evidências e poucos aplicativos voltados para treinamento de resistência incluíram estes tipos de metas.

Já Lewis e Wyatt (2014) destacaram a existência de estudos nos quais aplicativos e outros dispositivos móveis possam possuir potencial para auxiliar em tarefas médicas de rotina, incluindo a questão da educação do paciente e também o acesso a registros médicos. Contudo, ressaltam a importância desses aplicativos médicos apresentarem precisão e confiabilidade, principalmente quanto há tomadas de decisões com base em suas informações.

Outra questão importante levantada por Lewis e Wyatt (2014) é que alguns estudos relataram que há pouca ou nenhuma proximidade dos desenvolvedores com treinamento médico e, além disso, muitas vezes os médicos não estão envolvidos no processo de criação e desenvolvimento dos aplicativos, o que pode comprometer o uso pelo desconhecimento de questões de segurança, devido ao mau funcionamento ou mesmo pelo conteúdo inadequado.

Assim, Modave et al. (2015) sustentam em seu estudo a necessidade de criação de aplicativos que abordem o condicionamento físico baseados nas diretrizes do ACSM na prescrição dos exercícios. Além disso, citam que há uma ótima oportunidade no momento para que os desenvolvedores contribuam para o campo da saúde e atividades físicas.

Neste mesmo sentido, Knight et al. (2015) concluem que poucos aplicativos de atividade física são baseados em evidências científicas, necessitando que essa falha seja corrigida para possibilitar inclusive o uso clínico dessas possibilidades tecnológicas, melhorando os resultados em saúde e auxiliando o profissional. Por fim, destacam que utilizar ferramentas que possibilitem integração social (conexão e compartilhamentos, por exemplo) e recursos tecnológicos (conexão com recursos periféricos) podem potencializar os resultados visando aos usuários saudáveis. 
Aplicativos de smartphones e atividades físicas: contribuições e limitações

Renato Henrique Verzani • Adriane Beatriz de Souza Serapião

Assim, Lewis e Wyatt (2014) sugerem uma estrutura de risco genérica para auxiliar na busca por avaliação de riscos de aplicativos em determinados contextos. Eles citam duas dimensões de risco, sendo a primeira a probabilidade de um evento ocorrer e causar danos e a segunda a gravidade do dano ocorrido. Contudo, os fatores de risco podem ser reduzidos a partir de uma regulamentação adequada, sendo que, por outro lado, as questões contextuais podem carecer de uma educação formal e conscientização dos usuários.

Uma das maiores ameaças à segurança dos pacientes citada por Lewis e Wyatt, (2014) a partir do uso dos aplicativos médicos, seria o conhecimento inadequado/insuficiente de profissionais e pacientes sobre o risco, sendo que muitas vezes as próprias ações dos usuários nesses aplicativos podem ocasionar danos, ao invés do aplicativo em si. Assim, consideram que, para minimizar os riscos, seria interessante desenvolver estratégias educacionais para ampliar a conscientização sobre questões de segurança do paciente e dos riscos envolvidos na utilização incorreta dos aplicativos. Essa lógica sugerida pelos autores para os aplicativos médicos e seus profissionais também poderia ser considerada pelos profissionais de Educação Física para os aplicativos envolvidos com sua atividade.

\section{Considerações finais}

Os dados apresentados permitem novos olhares na busca por um estilo de vida mais ativo e saudável, além da contribuição para o acompanhamento de condições crônicas e o aumento da consciência a partir do automonitoramento, promovendo alterações tanto da perspectiva dos usuários como dos profissionais da área. Considerando os problemas relacionados à inatividade física em todo o mundo, é imprescindível traçar estratégias e apropriar-se das potencialidades dos novos recursos que conduzam ao aumento da participação regular das pessoas em atividades físicas. 
Aplicativos de smartphones e atividades físicas: contribuições e limitações

Renato Henrique Verzani • Adriane Beatriz de Souza Serapião

Assim, esses avanços tecnológicos têm sido vistos como grandes oportunidades, permitindo a adoção e combinação de diversas estratégias que têm apresentado resultados promissores, como automonitoramento, feedbacks, dentre outros, ampliando o engajamento dos usuários e refletindo na saúde. Contudo, é importante enfatizar que essa promoção das atividades físicas foi mais eficiente com intervenções que vão além de apenas disponibilizar os aplicativos. Por outro lado, cabe também atenção especial e alguns cuidados quanto a utilização desses recursos dos aplicativos, como imprecisões de alguns sensores, respeito a diretrizes disponíveis sobre atividades físicas, dentre outros aspectos apresentados. A orientação de especialistas da área na criação e desenvolvimento dos aplicativos Ihes ofereceriam maior confiabilidade.

Portanto, na busca dos profissionais por um maior monitoramento que possa desencadear em melhorias na saúde de seus clientes ou pacientes, há um grande potencial na inclusão dos aplicativos. Tendo em mãos todas as informações coletadas, aprimora-se o planejamento e a busca por resultados. Além disso, essas ferramentas também podem contribuir para a motivação e a avaliação dos usuários que não têm tanto acesso aos serviços de saúde (HIGGINS, 2016).

Sendo assim, o potencial e a quantidade de recursos desses aplicativos são inúmeros, cabendo aos profissionais estarem atentos tanto aos benefícios quanto aos riscos e cuidados na sua utilização, bem como promoverem a conscientização de seus alunos e dos usuários quanto a esses fatores. É imprescindível que os profissionais estejam antenados com os novos avanços tecnológicos e deem suas contribuições no seu desenvolvimento e aprimoramento, trazendo não somente maior fidedignidade aos resultados coletados, como também viabilizando sua utilização de forma mais segura. Conforme demonstrado, aliar diversas estratégias que mantenham os usuários em rotinas de atividades físicas ajuda a combater os riscos associados ao sedentarismo e contribui para melhores resultados na saúde pública. A consolidação dessas tecnologias permite uma expansão dos horizontes na perspectiva da 
Aplicativos de smartphones e atividades físicas: contribuições e limitações

Renato Henrique Verzani • Adriane Beatriz de Souza Serapião

prática profissional, aumentando as expectativas quanto a reais mudanças na realidade vivenciada e trazendo melhores resultados nas prescrições de exercícios físicos para os mais diversos públicos.

\section{Referências}

ANDERSEN, L.B.; MOTA, J.; DI PIETRO, L. Update on the global pandemic of physical inactivity. Lancet, v. 388, n. 10051, p. 12556, 2016. [doi: 10.1016/S0140-6736(16)30960-6].

AREM, H.; MOORE, S. C.; PATEL, A.; HARTGE, P.; BERRINGTON DE GONZALEZ, A.; VISVANATHAN, K.; CAMPBELL, P. T.; FREEDMAN, M.; WEIDERPASS, E.; ADAMI, H. O.; LINET, M. S.; LEE, I.

M.; MATTHEWS, C. E. Leisure time physical activity and mortality: a detailed pooled analysis of the dose-response relationship.

JAMA Intern Med, v. 175, n. 6, p. 959-67, 2015. [doi: 10.1001/ jamainternmed.2015.0533].

ARONI, A.; CASTILLO, E.; SOUSA, C.; MACHADO, A. A.; FILHO, E.; TENENBAUM, G. Smartphone Applications Used for Initiating and Maintaining Physical Activity: An Exploratory Analysis. Revista de Psicología del Deporte, v. 27, suppl. 1, p. 89-95, 2017.

BIRKHOFF, S. D.; MORIARTY, H. Interventions using smartphone health apps across various populations: an integrative review of literature. Journal of Informatics Nursing, v. 1, n. 1, p. 13-24, 2016.

BIRKHOFF, S. D.; SMELTZER, S. C. Perceptions of Smartphone User-Centered Mobile Health Tracking Apps Across Various Chronic Illness Populations: An Integrative Review. J Nurs Scholarsh, v. 49, n. 4, p. 371-378, 2017. [doi: 10.1111/jnu.12298.]. BISWAS, A.; OH, P.I.; FAULKNER, G.E.; BAJAJ, R. R.; SILVER, M. A.; MITCHELL, M. S.; ALTER, D. A. Sedentary time and its association with risk for disease incidence, mortality, and hospitalization in adults: a systematic review and meta-analysis. Ann Intern Med, v. 162, n. 2, p. 123-32, 2015. [doi: 10.7326/M14-1651]. 
Aplicativos de smartphones e atividades físicas: contribuições e limitações

Renato Henrique Verzani • Adriane Beatriz de Souza Serapião

BORT-ROIG, J.; GILSON, N. D.; PUIG-RIBERA, A.; CONTRERAS, R.S.; TROST, S. G. Measuring and influencing physical activity with smartphone technology: a systematic review. Sports Med, v. 44, n. 5, p. 671-686, 2014. [doi: 10.1007/s40279-014-0142-5].

BOULOS, M. N.; WHEELER, S.; TAVARES, C.; JONES, R. How smartphones are changing the face of mobile and participatory healthcare: an overview, with example from eCAALYX. BioMed. Eng. OnLine, v. 10, p. 1-14, 2011. [doi:10.1186/1475-925X-10-24].

DEMONTE, M. L. B. Design para persuasão móvel: promoção e controle de hábitos saudáveis. 2016. 96f. Dissertação (Mestrado em Design). Universidade de Brasília, Brasília, 2016.

DIREITO, A.; PFAEFFLI DALE, L.; SHIELDS, E.; DOBSON, R.;

WHITTAKER, R.; MADDISON, R. Do physical activity and dietary smartphone applications incorporate evidence based behaviour change techniques? BMC Public Health, v. 14, p. 1-7, 2014. [doi: 10.1186/1471-2458-14-646].

EKELUND, U.; STEENE-JOHANNESSEN, J.; BROWN, W.J.;

FAGERLAND, M. W.; OWEN, N.; POWELL, K. E.; BAUMAN, A.; LEE, I. $M$. Does physical activity attenuate, or even eliminate, the detrimental association of sitting time with mortality? A harmonised meta-analysis of data from more than 1 million men and women. Lancet, v. 388, n. 10051, p. 1302-10, 2016. [doi: 10.1016/S01406736(16)30370-1].

ELIAS, C. S. R.; SILVA, L. A.; MARTINS, M. T. S. L.; RAMOS, N. A. P.; SOUZA, M. G. G.; HIPÓLITO, R. L. Quando chega o fim? Uma revisão narrativa sobre terminalidade do período escolar para alunos deficientes mentais. SMAD: Rev. Eletrônica Saúde Mental Álcool Drog., v. 8, n. 1, p. 48-53, 2012.

GABBIADINI, A.; GREITEMEYER, T. Fitness mobile apps positively affect attitudes, perceived behavioral control and physical activities. J Sports Med Phys Fitness, v. 59, n. 3, p. 407-14, 2019. [doi: 10.23736/S0022-4707.18.08260-9]. 
Aplicativos de smartphones e atividades físicas: contribuições e limitações

Renato Henrique Verzani • Adriane Beatriz de Souza Serapião

GAL, R.; MAY, A. M.; VAN OVERMEEREN, E. J.; SIMONS, M.; MONNINKHOF, E. M. The Effect of Physical Activity Interventions Comprising Wearables and Smartphone Applications on Physical Activity: a Systematic Review and Metaanalysis, Sports Med Open, v. 4, n. 1, p. 1-15, 2018. [doi: 10.1186/ s40798-018-0157-9].

GRAND VIEW RESEARCH. mHealth Market Size Worth \$151.57 Billion By 2025, 2018. Disponível em: https://www.grandviewresearch.com/press-release/global-mhealth-market. Acesso em: 06 mar. 2019.

HALLAL, P. C.; ANDERSEN, L. B.; BULL, F. C.; GUTHOLD, R.; HASKELL, W.; EKELUND, U. Global physical activity levels: surveillance progress, pitfalls, and prospects. Lancet, v. 380, n. 9838, p. 247-257, 2012. [doi:10.1016/S0140-6736(12)60646-1].

HASMAN, L. An introduction to consumer health apps for the iPhone. J Consum Health Internet, v. 15, n. 4, p. 322-329, 2011. [doi: 10.1080/15398285.2011.623567]

HERRMANN, L. K.; KIM, J. The fitness of apps: a theory-based examination of mobile fitness app usage over 5 months. Mhealth, v. 3, p. 1-9, 2017. [doi: 10.21037/mhealth.2017.01.03].

HICKEY, A. M.; FREEDSON, P. S. Utility of consumer physical activity trackers as an intervention tool in cardiovascular disease prevention and treatment. Prog Cardiovasc Dis, v. 58, n. 6, p. 613-619, 2016. [doi:10.1016/j.pcad.2016.02.006].

HIGGINS, J. P. Smartphones applications for patients' health and fitness. Am J Med, v. 129, n. 1, p. 11-19, 2016. [doi: 10.1016/j. amjmed.2015.05.038].

JOSEPH, R. P.; DURANT, N. H.; BENITEZ, T. J.; PEKMEZI, D. W. Internet-based physical activity interventions. Am J Lifestyle Med, vol. 8, n. 1, p. 42-68, 2014. [doi: 10.1177/1559827613498059].

KNIGHT, E. Leveraging the Power of Social Media to Engage Patients and Achieve Treatment Outcomes. Crit Rev Phys 
Aplicativos de smartphones e atividades físicas: contribuições e limitações

Renato Henrique Verzani • Adriane Beatriz de Souza Serapião

Rehabil Med, v. 24, n. 3-4, p. 169-177, 2012. [doi: 10.1615/

CritRevPhysRehabilMed.2013007169].

KNIGHT, E.; STUCKEY, M. I.; PRAPAVESSIS, H.; PETRELLA, R. J. Public health guidelines for physical activity: is there an app for that? $A$ review of android and apple app stores. JMIR Mhealth Uhealth. v. 21, n. 3, e43, 2015. [doi: 10.2196/mhealth.4003].

LAMBOGLIA, C. M. G. F.; SILVA, C. A. B.; VASCONCELOS FILHO, J. E.; CARVALHO, L. M.; SILVA JUNIOR, F. V. I. O vilão se torna mocinho: uma perspectiva inovadora da utilização das tecnologias de entretenimento e comunicação para a promoção e práticas em saúde. In: SANTOS, Z. M. S. A.; FROTA, M. A.; MARTINS, A. B. T. (Org.). Tecnologias em saúde: da abordagem teórica a construção e aplicação no cenário do cuidado. 1 ed. Fortaleza: EdUECE, 2016. p. 43-63.

LEWIS, T. L.; WYATT, J. C. mHealth and mobile medical apps: a framework to Assess risk and promote safer use. J Med Internet Res, v. 16, n. 9, e210, 2014.

LOCKE E, LATHAM G. New directions in goal-setting theory. Curr Dir Psychol Sci, v. 15, n. 5, p. 265-268, 2006.

MATTHEWS, J.; WIN, K. T.; OINAS-KUKKONEN, H.; FREEMAN, M. Persuasive Technology in Mobile Applications Promoting Physical Activity: a Systematic Review. J Med Syst, v. 40, n. 3, p. 1-13, 2016. [doi: 10.1007/s10916-015-0425-x].

McCALLUM, C.; ROOKSBY, J.; GRAY, C. M. Evaluating the Impact of Physical Activity Apps and Wearables: Interdisciplinary Review. JMIR Mhealth and Uhealth, v. 6, p. 3, e58, 2018. [doi: 10.2196/ mhealth.9054].

MELO, D. A. T. Promoção da prática de exercício físico através de um jogo. 2017. 132f. Dissertação (Mestrado em Engenharia Informática). Instituto Superior de Engenharia do Porto, Porto, 2017. 
MIDDELWEERD, A.; MOLLEE, J. S.; VAN DER WAL, C. N.; BRUG, J.; TE VELDE, S. J. Apps to promote physical activity among adults: a review and content analysis. Int J Behav Nutr Phys Act, v. 11, p. 1-9, 2014. [doi: 10.1186/s12966-014-0097-9].

MODAVE, F.; BIAN, J.; LEAVITT, T.; BROMWELL, J.; HARRIS, III, C.; VINCENT, H. Low Quality of Free Coaching Apps With Respect to the American College of Sports Medicine Guidelines: A Review of Current Mobile Apps. JMIR Mhealth Uhealth, vol. 3, n. 3, e77, 2015. [doi:10.2196/mhealth.4669].

MURRAY, J. M.; BRENNAN, S. F.; FRENCH, D. P.; PATTERSON, C. C.; KEE, F.; HUNTER, R. F. Effectiveness of physical activity interventions in achieving behavior change maintenance in young and middle aged adults: a systematic review and meta-analysis.

Soc Sci Med, v. 192, p. 125-133, 2017. [doi: 0.1016/j.socscimed.2017.09.021].

NAKHASI, A.; SHEN, A. X.; PASSARELLA, R. J.; APPEL, L.

J.; ANDERSON, C. A. Online social networks that connect users to physical activity partners: a review and descriptive analysis. J Med Internet Res, v. 16, n. 6, e153, 2014. [doi: 10.2196/jmir.2674].

NICHOLSON, S. A user-centered theoretical framework for meaningful gamifcation. Paper presented at Games+Learning+Society 8.0, Madison, 2012.

PATEL, M. S.; ASCH, D.A.; VOLPP, K. G. Wearable Devices as Facilitators, not Drivers, of Health Behavior Change. JAMA, v. 313, n. 5, p. 459-460, 2015. [doi: 10.1001/jama.2014.14781].

RABIN, C.; BOCK, B. Desired features of smartphone applications promoting physical activity. Telemed J E Health, v. 17, n. 10, p. 801-3, 2011. [doi: 10.1089/tmj.2011.0055].

RECIO-RODRIGUEZ, J.I.; MARTIN-CANTERA, C.; GONZALEZ-VIEJO, N.; ARIETALEANIZBEASCOA, M. S.; SCHMOLLING-GUINOVART, Y.; MADERUELO-FERNANDEZ, J. A.; PÉREZ-ARECHAEDERRA, D.; RODRIGUEZ-SANCHEZ, E.; GÓMEZ-MARCOS, M. A.; GARCÍAORTIZ, L.; EVIDENT GROUP. Effectiveness of a smartphone application for improving healthy lifestyles, a randomized clinical trial 
Aplicativos de smartphones e atividades físicas: contribuições e limitações

Renato Henrique Verzani • Adriane Beatriz de Souza Serapião

(EVIDENT II): study protocol. BMC Public Health, v. 14, p. 1-13, 2014. [doi: 10.1186/1471-2458-14-254].

REINER, M.; NIERMANN, C.; JEKAUC, D.; WOLL, A. Long-term health benefits of physical activity: A systematic review of longitudinal studies. BMC Public Health, v. 13, p. 1-9, 2013. [doi: 10.1186/1471-2458-13-813].

REZENDE, L. F.; RODRIGUES, L. M.; REY-LÓPEZ, J.; MATSUDO, V.; LUIZ, O. C. Sedentary behavior and health outcomes: An overview of systematic reviews. PLoS One, v. 21, n. 9, e105620, 2014. [doi: 10.1371/journal.pone.0105620].

ROTHER, E. T. Revisão sistemática X revisão narrativa. Acta Paul Enferm, v. 20, n. 2, p. v-vi, 2007.

RYAN, R. M.; DECl, E. L. Intrinsic and extrinsic motivations: classic definitions and new directions. Contemporary Educational psychology, v. 25, n. 1, p. 54-67, jan. 2000a.

RYAN, R. M.; DECl, E. L. Self-Determination Theory and the Facilitation of Intrinsic Motivation, Social Development, and WellBeing. The American Psychologist, v. 55, n. 1, p. 68-78, $2000 \mathrm{~b}$. SAMA, P. R.; EAPEN, Z. J.; WEINFURT, K. P.; SHAH, B. R.; SCHULMAN, K. A. An evaluation of mobile health application tools. JMIR MHealth Uhealth, v. 2, n. 2, e19, 2014. [doi: 10.2196/ mhealth.3088].

SAMDAL, G. B.; EIDE, G. E.; BARTH, T.; WILLIAMS, G.; MELAND, E. Effective behavior change techniques for physical activity and healthy eating in overweight and obese adults; systematic review and meta-regression analyses. Int J Behav Nutr Phys Act, v. 14, n. 1, p. 1-14, 2017. [doi: 10.1186/s12966-017-0494-y].

SANDERS, J. P.; LOVEDAY, A.; PEARSON, N.; EDWARDSON, C.; YATES, T.; BIDDLE, S. J.; ESLIGER, D. W. Devices for self-monitoring sedentary time or physical activity: a scoping review. J Med Internet Res, v. 18, e90, 2016.[doi: 10.2196/jmir.5373].

SARKAR, U.; GOURLEY, G. I.; LYLES, C. R.; TIEU, L.; CLARITY, C.; NEWMARK, L.; SINGH, K.; BATES, D. W. Usability of commercially 
available mobile applications for diverse patients. J Gen Intern Med, v. 31, n. 12, p. 1417-1426, 2016.

SCHOEPPE, S.; ALLEY, S.; VAN LIPPEVELDE, W.; BRAY, N. A.; WILLIAMS, S. L.; DUNCAN, M. J.; VANDELANOTTE, C. Efficacy of interventions that use apps to improve diet, physical activity and sedentary behaviour: a systematic review. Int J Behav Nutr Phys Act, v. 13, n. 1, p. 1-26, 2016. [doi:10.1186/s12966-016-0454-y].

SHUGER, S. L.; BARRY, V. W.; SUI, X.; HAND, G. A.; WILCOX, S.; MERIWETHER, R. A.; HARDIN, J. W.; BLAIR, S. N. Electronic feedback in a diet- and physical activity-based lifestyle intervention for weight loss: a randomized controlled trial. Int J Behav Nutr Phys Activ, v. 8, n. 1, p. 1-8, 2011. [doi: 10.1186/1479-5868-8-41]. SPITTAELS, H.; DE BOURDEAUDHUIJ, I.; VANDELANOTTE, C. Evaluation of a website-delivered computer-tailored intervention for increasing physical activity in the general population. Prev Med, v. 44, n. 3, p. 209-217, 2007.

STRAGIER, J.; ABEELE, M. V.; MAREZ, L. Recreational athletes' running motivations as predictors of their use of online fitness community features. Behavior \& Information Tecnology. v. 37, n. 8, p. 815-827, 2018. [https://doi.org/10.1080/014492 9X.2018.1484516].

TURNER-McGRIEVY, G. M.; BEETS, M. W.; MOORE, J. B.; KACZYNSKI, A. T.; BARR-ANDERSON, D. J.; TATE, D.F. Comparison of traditional versus mobile app self-monitoring of physical activity and dietary intake among overweight adults participating in an mHealth weight loss program. J am med Inform Assoc, v. 20, n. 3, p. 513518, 2013. [doi: 10.1136/amiajnl-2012-001510].

VOSGERAU, D. S. R.; ROMANOWSKI, J. P. Estudos de revisão: implicações conceituais e metodológicas. Rev. Diálogo Educ., v. 14, n. 41, p. 165-189, 2014. [doi: 10.7213/dialogo.educ.14.041.DS08].

WANG, Q.; EGELANDSDAL, B.; ANDAM, G. V.; ALMLI, V. L.; OOSTINDJER, M. Diet and Physical Activity Apps: Perceived Effectiveness by App Users. JMIR mHealth uHealth, v. 4, n. 2, p. 1-14, 2016. [doi: 10.2196/mhealth.5114]. 
Aplicativos de smartphones e atividades físicas: contribuições e limitações

Renato Henrique Verzani • Adriane Beatriz de Souza Serapião

WERBACH, K.; HUNTER, D. For the Win: How Game Thinking Can Revolutionize Your Business. Philadelphia: Wharton Digital Press, 2012.

WHO. Physical activity. 2018. Disponível em: https://www.who. int/en/news-room/fact-sheets/detail/physical-activity. Acesso em: 10 jul. 2019.

WINTERSTEIN, P. J.; VENDITTI JR, R. A motivação para as práticas corporais e para o esporte. In: DE ROSE Jr., D. et al. Esporte e Atividade Física na Infância e na Adolescência. 2.a. ed., Porto Alegre: Artmed, 2009. p. 115-136.

WU, W.; DASGUPTA, S.; RAMIREZ, E. E.; PETERSON, C.; NORMAN G. J. Classification accuracies of physical activities using smartphone motion sensors. J Med Internet Res, v. 14, n. 5, e130, 2012. [doi: 10.2196/jmir.2208].

YUAN, S.; MA, W.; KANTHAWALA, S.; PENG, W. Keep Using My Health Apps: Discover Users' Perception of Health and Fitness Apps with the UTAUT2 Model. Telemed J E Health, v. 21, n. 9, p. 735-41, 2015. [doi: 10.1089/tmj.2014.0148].

\section{Financiamento}

Esta pesquisa contou com apoio financeiro da Coordenação de Aperfeiçoamento de Pessoal de Nível Superior (CAPES)

\section{Publisher}

Universidade Federal de Goiás. Faculdade de Educação Física e Dança. Publicação no Portal de Periódicos UFG. As ideias expressadas neste artigo são de responsabilidade de seus autores, não representando, necessariamente, a opinião dos editores ou da universidade. 